\title{
Multiagent-Based Educational Environment for Dependents
}

\author{
Antonia Macarro, Alberto Pedrero, and Juan A. Fraile \\ Pontifical University of Salamanca, Compañía 5, 37002 Salamanca, Spain \\ \{mmacarroal, apedreroes, jafraileni\} @upsa.es
}

\begin{abstract}
This paper presents a multiagent architecture that facilitates active learning in educational environments for dependents. The multiagent architecture incorporates agents that can be executed on mobile devices and that facilitate language learning in an ubiquitous way. Moreover, the agents are specifically designed to provide advanced interfaces for elderly and dependent people. The architecture has been tested in a real environment and the preliminary results obtained are presented within this paper.
\end{abstract}

Keywords: Ambient Intelligence, language learning, Multiagent systems, elderly people.

\section{Introduction}

Ambient Intelligence evolves from the ubiquitous computing [4], and constitutes the most promising technological approach to meet the challenge of developing strategies for early detection and prevention of problems in dependency environments [17]. One of the segments of the population who will benefit with the advent of systems based on Ambient Intelligence will be the elderly and people with disabilities [8], contributing to improve their quality of life [10].

Different authors [3] [9] [18] consider that in the near future, the institutions for health care will be provided with intelligent Systems capable to easily interact with the humans. It is necessary to improve the supply of services, as well as the way to offer them. Trends situate the user surrounded of technology that facilitates new and personalized services. Multiagent systems and architectures based on mobile devices, have recently been explored as a system of interaction with the elderly and dependent [1]. These systems can provide support in the daily lives of dependent people [20], providing a cognitive and physical support for the assisted person [2]. They can also provide mechanisms for establishing new strategies for learning interaction, which greatly facilitates the teaching, particularly languages learning.

The aim of this work is to provide a methodology, on the basis of the Ambient Intelligence (AmI) [5] [15], for formal teaching of languages oriented to dependent people. We propose the combination of new technologies along with the traditional teaching. In this way it will be possible to combine the advantages of the face to face teaching with the advantages of distance learning. It will be necessary to upgrade the systems of evaluation/accreditation to assess the knowledge or skills acquired during 
the learning process. To achieve this objective, we propose the use artificial intelligence techniques, intelligent agents and wireless communications.

The rest of the paper is structured as follows: Next section introduces the problem that motivates most of this research. Section 3 presents the multiagent architecture proposed to resolve the problem. Section 4 describes a case study to test the proposal and, finally, Section 5 presents the results and conclusions obtained.

\section{Ambient Intelligence in Dependent Educational Environments}

There is an ever growing need to supply constant care and support to the disabled and elderly and the drive to find more effective ways to provide such care has become a major challenge for the scientific community [4]. During the last three decades the number of Europeans over 60 years old has risen by about $50 \%$. Today they represent more than $25 \%$ of the population and it is estimated that in 20 years this percentage will rise to one third of the population, meaning 100 millions of citizens [4]. In the USA, people over 65 years old are the fastest growing segment of the population [1] and it is expected that in 2020 they will represent about 1 of 6 citizens totaling 69 million by 2030 . Furthermore, over $20 \%$ of people over 85 years old have a limited capacity for independent living, requiring continuous monitoring and daily care [2]. Some estimations of the World Health Organization show that in 2025 there will be more than 1000 million people aged over 60 in the world, so if this trend continues, by 2050 will be double, with about the $80 \%$ concentrated in developed countries [18].

Education is the cornerstone of any society and it is the base of most of the values and characteristics of that society. The new knowledge society offers significant opportunities for AmI applications, especially in the fields of education and learning [17]. The new communication technologies propose a new paradigm focused on integrating learning techniques based on active learning (learning by doing things, exchange of information with other users and the sharing of resources), with techniques based on passive learning (learning by seeing and hearing, Montessori, etc.) [7]. While the traditional paradigm, based on a model focused on face to face education, sets as fundamental teaching method the role of the teachers and their knowledge, the paradigm based on a learning model highlights the role of the students. In this second paradigm the students play an active role, and build, according to a personalized action plan, their own knowledge. Moreover, they can establish their own work rhythm and style. The active methodology proposes learning with all senses (sight, hearing, touch, smell and taste), learn through all possible methods (school, networking, etc.), and have access to knowledge without space or time restrictions (anywhere and at any time).

There are different studies that have used the Ambient Intelligence to facilitate learning. In [4], Bomsdorf shows the need to adapt intelligent environments to changes depending on the educational context and the characteristics of users. Morken et al. [18] analyze the characteristics of intelligent environments for learning. They focus on the role of mobility in educational environments and the role that acquire the mobile devices. Naismith et al. [19] conducted a detailed study describing the role of mobile devices in education, analyzing the characteristics of the devices and their capacity for learning in educational environments. All these approaches are 
focused on the role of learning in Ambient Intelligence environments, but none of them is oriented on learning for dependents or elderly people. The following section presents a multiagent architecture that facilitates learning methodology using an active through mobile devices.

\section{Multiagent-Based Intelligent Environment for Language Education}

Information technologies can notably improve the learning process in educational environments, providing the users with new technological resources [7]. At present there are various tools to facilitate the active learning, such as forums, wikis, email, chat, virtual campuses, and so on. However, none of them is focused to language learning for elderly people or dependents. This paper presents a multiagent architecture specially designed to facilitate active learning in dependence environments. Agents can be characterized through their capacities in areas such as autonomy, reactivity, proactivity, social abilities, reasoning, learning and mobility [12] [21], which make them particularly suitable for use in the design and development of intelligent environments for dependency. That way, each student can have a personal agent, able to adapt to his personal needs taking into account the characteristics of his profile. In addition, each teacher has a personal agent that facilitates his interaction with students. The outline of the proposed architecture is presented in Figure 1.

In Figure 1 it is possible to see the four types of agents defined within the architecture: a student agent, a professor agent, an evaluator agent and a manager agent. The student and professor agents can be run on mobile devices, while the evaluator and manager agents run on a central computer. In the following paragraph, the main characteristics of the agent types are presented:

- Student Agent. It is a type of interface agent that allows a student to interact with the system. The student agents adapt to the needs of the human students, taking into consideration their profiles and customizing their access to the system.

- Professor Agent. It is a type of interface agent that allows a teacher to interact with the multiagent system. Through this agent a teacher is able to establish tasks for the students, and keep track of the personal development of the students

- Evaluator Agent. This agent is responsible for evaluating the work done by the students and allows progress on the level of difficulty. It is the agent that evaluates the level of learning acquired by the students. Moreover, it is in charged of statistics and reports periodically delivered to the teachers.

- Manager Agent. This agent is responsible for all management tasks within the system. These tasks include communications between the other agents in the multiagent system, assignation of tasks to students, or the creation of reports to be delivered to the professors. The allocation of tasks is based on past experiences, using a case-based reasoning system [9], so that the agent is able to learn and perform a more efficient assignation of tasks to students. Case-based reasoning is a technique in which past experiences are used to 
solve new problems. This technique has been successfully used for the assignation of tasks on previous works focused on planning the working day of nurses and doctors in geriatric residences [5] [10] [11], or planning guidance routes in shopping malls [4].

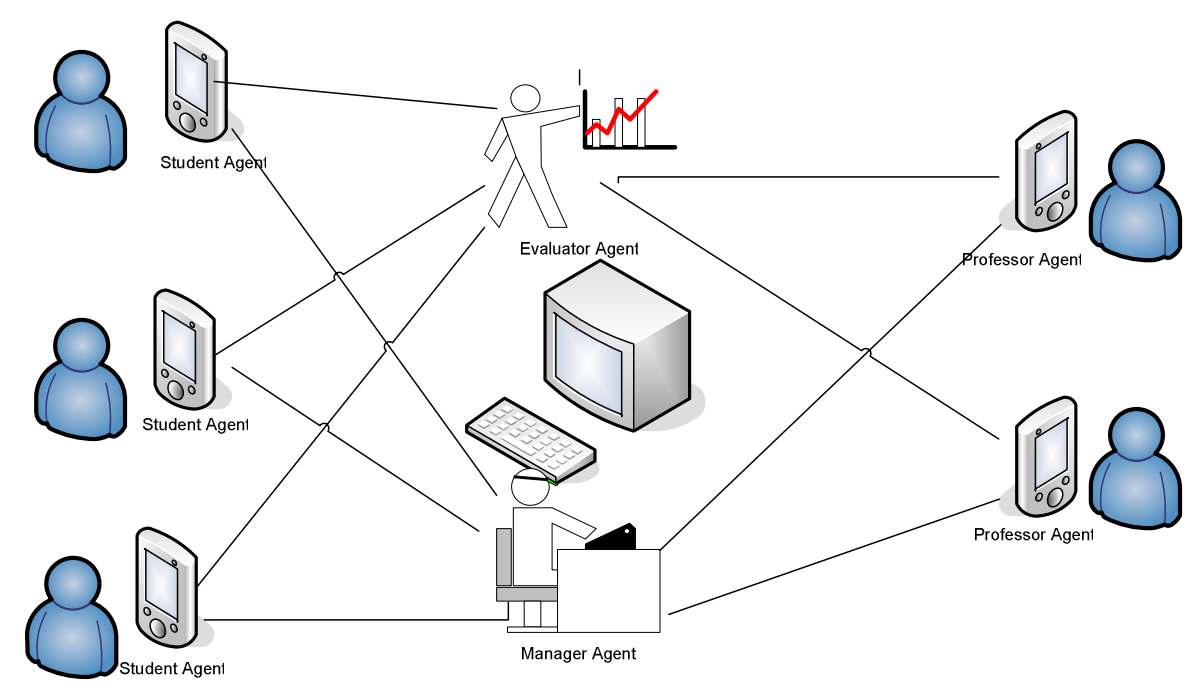

Fig. 1. Multiagent architecture for educational environments

The proposed architecture has great capacity for learning and adaptation to the user needs. Some works [14], which proposes an e-mail system adapted to elderly people without experience in the use of computers, show that not all users have the same difficulties. Even when an application was developed specifically for them, may require different versions for different users depending on their characteristics. The customization of the tasks and the interface can solve this problem because it allows different versions depending on the needs and particularities of each user [18].

One of the advantages of the architecture proposed in this paper is the ability of the agents to communicate through wireless channels, which facilitate independence of location or time constraints. The professor and student agents can be executed in mobile devices, and communicate using Wi-Fi, Bluetooth, SMS or MMS technologies. In this way, the students can use the learning system at their homes regardless of timetables. Access to services is independent of the terminal used and the use of services is simple and homogeneous, allowing a rapid assimilation by the user. In addition, users can receive fully customized services.

\section{Case Study: Learning Languages at the Universidad de la Experiencia}

Concern about the education of elderly people has led the universities to set up, under different names, studies for elderly people. These initiatives began to be put into 
practice in Spain in the early 90's, and nowadays all the Spanish universities have an educational program for elderly. In general, elderly people are inexperienced in the use of the Internet, finding problems to understand and use some of the conventions used in user interfaces: double-clicking with the mouse, movements between text fields to be filled in, bar displacement [16]. Moreover, the problems are common vision [15], cognitive problems [10], manual dexterity [20]. In order to benefit this group from the advantages of the digital environment, it is necessary to provide new methods and techniques. The usability is a key factor, given that a difficult-to-use system will be probably rejected by the users, increasing the fear and, perhaps, a sense of frustration in the use of these technologies. The work of Marqui and Todman [18] presents the difficulties and negative experiences of the elderly people in the use of computers (mainly the anxiety it causes).

Among the agencies that investigate new proposals to improve the daily life of elderly and dependent people, we can find the European Educational Programs Autonomous Agency, whose mission is to manage the Spanish participation in the Lifelong Learning Program (PAP) of the European Union. Within the adult education program is included the Grundtvig program. Encourage learning throughout life has become a political priority for all European countries. Language training for dependents is common and widely accepted. In this way, under the frame of the Grundtvig program, we defined a case study at the University of the Experience at the Pontifical University of Salamanca. To encourage the active learning and the autonomy of the students, we designed a series of tests that can be completed in a simple way through mobile phones. The case study, that involved 20 individuals, allowed us to evaluate the proposed methodology, and more concretely the improvement of the knowledge acquired in French language learning. Specifically, we developed questions with multiple options (A B C D) presented in groups of 10 to each student. If the success obtained exceeded the $80 \%$, the student agreed to a higher level of difficulty. Moreover, at the same time the user chooses the option at the mobile phone, a microphone system records the phonetic. If the pronunciation is correct, the system recognizes the improvement and the user can advance to the next phase. In order to highly facilitate the user interaction with the multiagent system, we developed simple and intuitive interfaces. 5 tests were conducted at each of the students. Each test had a maximum duration of 60 minutes.

\section{Results and Conclusions}

This paper has presented a methodology for teaching languages to dependents, using techniques of Ambient Intelligence. The new multi-architecture presented in this paper provides an active learning through simple and intuitive interfaces, installed on mobile devices. This requires the integration of intelligent agents with innovative strategies of teaching languages and mobile devices. In this way we have obtained:

- An active method of teaching and learning of languages for dependents and elderly people.

- An interaction system based on the AmI paradigm for language education. 
- The obtained language learning system was adapted to be executed on mobile devices, facilitating the adaptation to the needs of the dependent and elderly people.

- The multiagent architecture presented in this work has been tested by means of the case study presented in Section 4.

Table 1 shows the average number of hits in each of the five tests of the case study, and the average time to complete the 10 questions per test for each of the students at the University of Experience. As shown in Table 1, in the first test, the students had low rates of success and the time spent completing them was greater. As students learnt, the success rate increased and the time to complete tests decreased.

Figure 2 shows the results of the five tests performed in the case study presented in Section 4. Figure 2 shows the evolution of the successes of the students in the tests. As can be seen, the number of successes was lower in the early tests, and increased as new tests were performed. The horizontal axis represents the time (the tests), and the vertical axis the number of successes along the time. It is usual to find errors at the beginning of a new task. In the later stages the error rate decreased, but also the learned new subjects, reaching a plateau. The task of learning for the French language presented in the case study is steep at the beginning and then increasingly flat. This means that at the beginning made great progress, but after a while acquiring new knowledge is more difficult.

Table 1. Average success rate and time (in minutes) for the tests in the case study

\begin{tabular}{lcc}
\hline & Success Rate & Time \\
\hline Test 1 & 32 & 51 \\
Test 2 & 43 & 47 \\
Test 3 & 56 & 42 \\
Test 4 & 77 & 38 \\
Test 5 & 85 & 34 \\
\hline
\end{tabular}

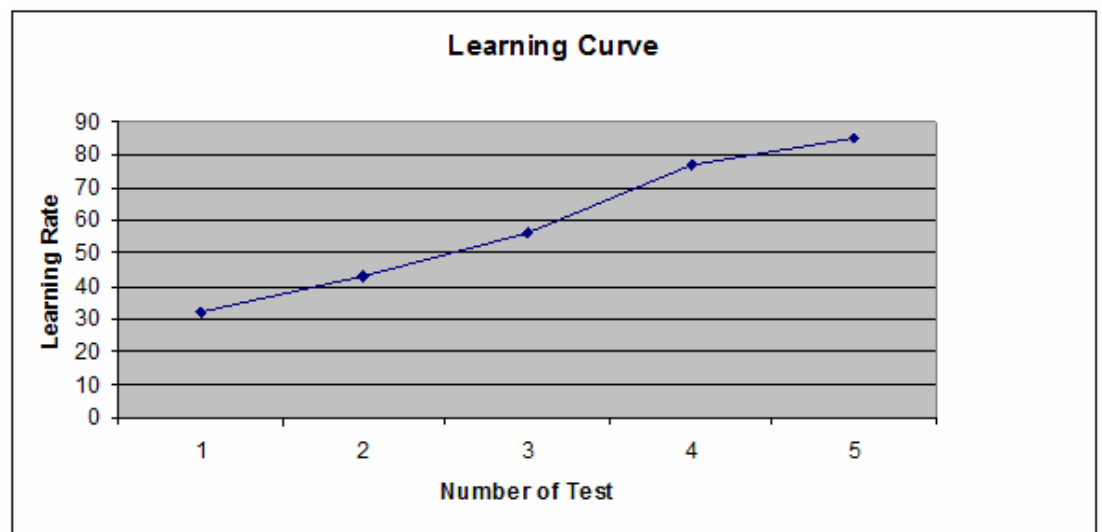

Fig. 2. Learning curve for the elderly students at the Universidad de la Experiencia. The curve shows the average learning rate for each of the five tests performed. 
This paper has presented a multiagent architecture that facilitates languages learning to elderly people. The preliminary results obtained in a case study in a real scenario are promising, and let us conclude that our proposal can be useful in educational environments. However, there are still certain aspects to improve. Unless the users have remarked the usability of the system, it is necessary to design more efficient interfaces, facilitating the interaction to disabled people. Moreover, it is necessary to evaluate the architecture in different environments and for different users. Finally, it would be useful to take into account the possibility of creating agent based virtual organizations, facilitating the simulation of situations and enabling the detection of key aspects for optimum learning.

Acknowledgments. This work has been supported by the MOVENTE project, founded by Fundación Caja Duero.

\section{References}

1. Anastasopoulos, M., Niebuhr, D., Bartelt, C., Koch, J., Rausch, A.: Towards a Reference Middleware Architecture for Ambient Intelligence Systems. In: ACM Conference on Object-Oriented Programming, Systems, Languages, and Applications (2005)

2. Angulo, C., Tellez, R.: Distributed Intelligence for smart home appliances. Tendencias de la minería de datos en España. Red Española de Minería de Datos. Barcelona, España (2004)

3. Bajo, J., Molina, J.M., Corchado, J.M.: Ubiquitous computing for mobile environments. In: Issues in Multi-Agent Systems: The AgentCities.ES Experience. Whitestein Series in Software Agent Technologies and Autonomic Computing, pp. 33-58. Birkhäuser, Basel (2007)

4. Bajo, J., Corchado, J.M., de Paz, Y., de Paz, J.F., Rodríguez, S., Martín, A., Abraham, A.: SHOMAS: Intelligent Guidance and Suggestions in Shopping Centres. Applied Soft Computing 9(2), 851-862 (2009)

5. Bajo, J., de Paz, J.F., de Paz, Y., Corchado, J.M.: Integrating Case-based Planning and RPTW Neural Networks to Construct an Intelligent Environment for Health Care. Expert Systems with Applications 36(3) part 2, 5844-5858 (2009)

6. Bomsdorf, B.: Adaptation of Learning Spaces: Supporting Ubiquitous Learning in Higher Distance Education. In: Davies, N., Kirste, T., Schumann, H. (eds.) Dagstuhl Seminar Proceedings. Mobile Computing and Ambient Intelligence: The Challenge of Multimedia, Dagstuhl, Germany (2005)

7. Brown, T.H.: Beyond constructivism: Exploring future learning paradigms. In: Education Today, issue 2 of 2005, Aries Publishing Company, Thames, New Zealand (2005)

8. Carretero, N., Bermejo, A.B.: Inteligencia Ambiental. CEDITEC: Centro de Difusión de Tecnologías, Universidad Politécnica de Madrid, España (2005)

9. Corchado, J.M., Laza, R.: Constructing Deliberative Agents with Case-based Reasoning Technology. International Journal of Intelligent Systems 18(12), 1227-1241 (2003)

10. Corchado, J.M., Bajo, J., Abraham, A.: GERAmI: Improving the delivery of health care. IEEE Intelligent Systems 23(2), 19-25 (2008)

11. Corchado, J.M., Bajo, J., de Paz, Y., Tapia, D.: Intelligent Environment for Monitoring Alzheimer Patients, Agent Technology for Health Care. Decision Support Systems 34(2), 382-396 (2008) 
12. Corchado, J.M., Glez-Bedia, J., de Paz, Y., Bajo, J., de Paz, J.F.: Replanning mechanism for deliberative agents in dynamic changing environments. Computational Intelligence 24(2), 77-107 (2008)

13. Czaja, S.J., Lee, C.C.: Designing computer systems for older adults. In: Jacko, J.A., Sears, J. (eds.) The Human-Computer Interaction Handbook: Fundamentals, Evolving Technologies and Emerging Applications, pp. 413-427. Lawrence Erlbaum, Mahwah (2003)

14. Dickinson, A., Newell, A.F., Smith, M.J., Hill, R.L.: Introducing the Internet to the over60s: developing an email system for older novice computer users. Interacting with Computers 17, 621-642 (2005)

15. Echt, K.V.: Designing web-based health information for older adults: visual considerations and design directives. In: Morrell, R.W. (ed.) Older adults, Health information and the World Wide Web, pp. 61-87. Lawrence Erlbaum Associates, Mahwah (2002)

16. Ellis, R.D., Kurniawan, S.H.: Increasing the usability of online information for older users: a case study in participatory design. International Journal of Human-Computer Interaction 2(12), 263-276 (2000)

17. Friedewald, M., Da Costa, O.: Science and Technology Roadmapping: Ambient Intelligence in Everyday Life (AmI@Life). Working Paper. Institute for Prospective Technology Studies IPTS, Seville (2003)

18. Kurniawan, S.H., King, A., Evans, D.G., Blenkhorn, P.L.: Personalising web page presentation for older people. Interacting with computers 18, 457-477 (2006)

19. Naismith, L., Lonsdale, P., Vavoula, G., Sharples, M.: Futurelab Literature Review in Mobile Technologies and Learning, Technical Report for Futurelab (2004),

http://www. futurelab.org.uk/research/reviews/reviews_11_and 12/11_01.htm

20. Ranganathan, V.K., Siemionow, V., Sahgal, V., Yue, G.H.: Effects of aging on hand function. Journal of the American Geriatrics Society 49, 1478-1484 (2001)

21. Wooldridge, M., Jennings, N.R.: Agent Theories, Architectures, and Languages: a Survey. In: Wooldridge, Jennings (eds.) Intelligent Agents, pp. 1-22. Springer, Heidelberg (1995) 